\title{
Which criteria characterize a health literate health care organization? - a scoping review on organizational health literacy
}

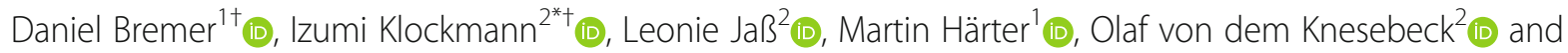
Daniel Lüdecke²

\begin{abstract}
Background: Organizational health literacy $(\mathrm{OHL})$ aims to respond to the health literacy needs of patients by improving health information and services and making them easier to understand, access, and apply. This scoping review primarily maps criteria characterizing health literate health care organizations. Secondary outcomes are the concepts and terminologies underlying these criteria as well as instruments to measure them.

Methods: The review was carried out following the JBI Manual on scoping reviews. The databases CINAHL, Cochrane Library, JSTOR, PsycINFO, PubMed, Web of Science Core Collection, and Wiley Online Library were searched in July 2020. Three researchers screened the records and extracted the data. The results were synthesized systematically and descriptively.

Results: The literature search resulted in 639 records. After removing duplicates, screening by title and abstract, and assessing full-texts for eligibility, the scoping review included 60 publications. Criteria for OHL were extracted and assigned to six main categories (with 25 subcategories). The most prevalent topic of organizational health literacy refers to communication with service users. Exemplary criteria regarding this main category are the education and information of service users, work on easy-to-understand written materials as well as oral exchange, and verifying understanding. The six main categories were defined as 1) communication with service users; 2) easy access \& navigation; 3) integration \& prioritization of $\mathrm{OHL}$; 4) assessments \& organizational development; 5) engagement \& support of service users, and 6) information \& qualification of staff. The criteria were based on various concepts and terminologies. Terminologies were categorized into four conceptual clusters: 1) health literacy in various social contexts; 2) health literate health care organization; 3) organizational behavior, and 4) communication in health care. 17 different assessment tools and instruments were identified. Only some of the toolkits and instruments were validated or tested in feasibility studies.
\end{abstract}

Conclusions: Organizational health literacy includes a significant number of distinct organizational criteria. The terminologies used in the OHL literature are heterogeneous based on a variety of concepts. A comprehensive, consensusbased conceptual framework on OHL is missing.

Keywords: Organizational health literacy, Health literacy responsiveness, Health care organizations, Health literacy, Organizational development, Patient-centered communication, Scoping review

\footnotetext{
* Correspondence: i.klockmann@uke.de

Daniel Bremer and Izumi Klockmann share first authorship.

${ }^{2}$ Department of Medical Sociology, Center for Health Care Research,

University Medical Center Hamburg-Eppendorf, Martinistr. 52, 20246

Hamburg, Germany

Full list of author information is available at the end of the article
}

(c) The Author(s). 2021 Open Access This article is licensed under a Creative Commons Attribution 4.0 International License, which permits use, sharing, adaptation, distribution and reproduction in any medium or format, as long as you give appropriate credit to the original author(s) and the source, provide a link to the Creative Commons licence, and indicate if changes were made. The images or other third party material in this article are included in the article's Creative Commons licence, unless indicated otherwise in a credit line to the material. If material is not included in the article's Creative Commons licence and your intended use is not permitted by statutory regulation or exceeds the permitted use, you will need to obtain permission directly from the copyright holder. To view a copy of this licence, visit http://creativecommons.org/licenses/by/4.0/ The Creative Commons Public Domain Dedication waiver (http://creativecommons.org/publicdomain/zero/1.0/) applies to the data made available in this article, unless otherwise stated in a credit line to the data. 


\section{Background}

Since the beginning of the 2000s, a new perspective on health literacy was introduced by focusing on the context, culture, and complexities of health care systems and health care organizations [1-3]. The idea of organizational health literacy (OHL) or health literacy responsiveness, defined as "the way in which services, organisations and systems make health information and resources available and accessible to people according to health literacy strengths and limitations" [4], was born. The concept arose from the acknowledgment that individual health literacy is considered an important issue for public health and health care [5]. Studies continue to show that large proportions of populations have insufficient or problematic health literacy $[6,7]$ and report even downward trends in health literacy over the last decade [8]. Limited health literacy is associated with a broad range of adverse health behaviors and health outcomes, e.g. causing poor self-efficacy regarding healthrelated issues, having less health-related knowledge, hindering medication adherence, generating higher rates of hospitalization, and provoking increased health care costs [9].

OHL focuses on the role of organizations to improve patient navigation through health care systems. It is consistent and important for health care organizations to respond to the health literacy needs of patients and to improve health information and services in order to make these more understandable and accessible [10-12]. Studies show that health care systems and their organizations do not react adequately to the existing and growing silent epidemic of insufficient health literacy [1316]. Over the last 15 years, a considerable amount of research on OHL has been conducted to promote and evaluate changes in various organizational contexts, e.g. hospitals [17-21] or in primary health care settings [2229].

Despite some specific literature reviews [20, 30-33], a consensus on criteria that characterize health literate health care organizations (HLHCOs) in different health care settings has not been reached. The reviews include some aspects of criteria that characterize HLHCOs, but they are limited to specific health care settings [20], focus on certain attributes of OHL $[20,30]$ and/or interventions [20,33], their evaluation [31] and implementation [32]. Furthermore, comprehensive and current information on the criteria's underlying concepts and terminologies, and on the instruments to measure these criteria, is expandable. Only a few studies investigated theories and concepts [30] and terminologies [34] of OHL. Both studies did not relate to the variety of criteria that characterize HLHCOs. The only review on instruments to measure OHL was performed in 2014 [35]. The study identified a large number of instruments to measure OHL, but focussed on only one database, certain attributes of HLHCOs [2], and quantitative measures.

This scoping review aims to first identify criteria that characterize HLHCOs across the literature. Secondly, it aims to describe concepts, terminologies, and instruments used to inform and measure these criteria. This review is part of the OHL-HAM study, a research project on OHL in the Hamburg Metropolitan Area (Germany) [36]. The aim of the study is to develop a consensus-based set of OHL criteria in consultation with various types of health care organizations.

\section{Materials and methods}

Since our research questions aim at the identification of key characteristics, key concepts, and terminologies as well as measurement instruments related to $\mathrm{OHL}$, a scoping review was conducted [37]. The scoping review followed the methodological framework of Arksey and O'Malley [38] and the guidelines of the JBI Manual for Evidence Synthesis [39, 40]. The reporting of this scoping review was based on the "Preferred Reporting Items for Systematic reviews and Meta-Analyses extension for Scoping Reviews (PRISMA-ScR) Checklist" [41] (additional file 1 "PRISMA-ScR Checklist"), the reporting guideline "Synthesis without meta-analysis (SWiM)" [42], and a study protocol including all preliminary specifications accessible on OSF Registries [43].

The study protocol included three further secondary research questions, which we also included in our data extraction and analysis. Due to the large amount of materials, we decided to omit detailed results concerning these three additional questions to avoid overburdening this manuscript. Instead, we included the respective results in the additional files section (additional file 2 "Process stages of becoming an HLHCO", 3 "Involved actors by type of organization", and 4 "Implemented interventions") and refer to them in the discussion.

\section{Data sources and search strategy}

After developing the research questions and performing a pilot run of literature search for configuring the search string, seven databases were used to gather the final records for our scoping review (3 July 2020). The final search was performed with CINAHL, Cochrane Library, JSTOR, PsycINFO, PubMed, Web of Science Core Collection, and Wiley Online Library. Using truncations and Boolean operators, the databases were searched for three sets of keywords referring to "organization", "health literacy/literate", and "criteria/criterion/attribut"/policy/ policies/guideline"/recommendation" " (additional file 5 "Search details"). Our search string followed the search strategy PICo (Population, Interest, Context) [44]. Search 
string and settings were adapted to the different databases.

\section{Eligibility criteria}

Literature was included that covered the context of health care provision (hospitals, health centers, physicians, etc.), and healthcare-related organizations (e.g. patient organizations, health insurances, physicians and psychotherapist associations, etc.) in any health care setting. Publications were included if they addressed OHL criteria. Literature reviews were used to identify additional records during the snowball process but were not extracted for data synthesis. No restrictions were defined regarding the source of information to prevent excluding specific types of evidence. No geographic or temporal restrictions were defined. Records published in languages other than German or English were excluded.

\section{Screening process}

A three-stage screening process was conducted to assess the relevance of the records. 1) Title and abstract screening: After merging the identified titles in a Citavi library (version 6), duplicates were removed and accessible abstracts were collected. Titles and abstracts of the remaining 301 records were screened by two pairs of researchers $(\mathrm{DB}, \mathrm{IK}$ (n records $=120)$, and DL, IK (n records $=181)$ ). The respective third researcher $(\mathrm{DB} / \mathrm{DL})$ was consulted for screening results deviating between the two initial researchers. Prior to the screening, two pilot screening phases, each covering $10 \%$ (randomly selected) of the records, were conducted by two researchers (DB, IK). 2) Full-text screening: After gathering all accessible full-texts, two researchers (DL, IK) conducted two pilot screening phases, each covering $10 \%$ (randomly selected) of the records. Subsequently, the two pairs of researchers conducted the screening of the full-texts, again consulting the respective third researcher (DB/DL) in case of deviations. 3) Snowballing: We examined the reference lists of the identified fulltext publications in order to identify other relevant sources of information.

\section{Data extraction}

The authors developed a standardized data extraction form, tailored to this scoping review. Before data charting, two reviewers (DB, IK) tested and adapted the extraction form to make sure that all relevant results were captured [38, 45, 46]. Data extraction and synthesis for the primary research question were done by one researcher (IK) and controlled by two researchers (DB, LJ). Data extraction for the other variables was done by three researchers $(\mathrm{DB}, \mathrm{IK}, \mathrm{LJ})$. In case of uncertainty, the research team was consulted. A formal risk of bias or quality assessment was neither necessary nor possible due to the character of a scoping review [37].

Using the standardized form, we collected information on 1) author, 2) year, 3) country, 4) type of publication, 5) type of study, and 6) group of participants, 7) criteria of OHL, 8) terminologies of OHL, 9) conceptual background of the terminologies, ${ }^{1}$ 10) assessment tools \& instruments, 11) methods of assessment, 12) processes to become an HLHCO, 13) type of organization, 14) actors, 15) service user, and 16) interventions to promote OHL.

To answer the primary research question, the records were screened for OHL criteria and related terms (e.g. attributes, health literacy responsiveness). The majority of publications structured the criteria into main (e.g. "communication") and subcategories (e.g. "communicates clearly"). In the case of multilevel lists of criteria, we only extracted the lowest list level available. This made the inductive process of clustering categories more independent from the original main categories, which were not extracted in such cases. When different publications referred to the same criteria, the criteria were only extracted once. For example, a group of publications [18, 47-52] referred to the "Ten Attributes of Health Literate Health Care Organizations" [2].

\section{Synthesis of results}

Our findings were synthesized descriptively and narratively to provide a systematic classification of OHL criteria as well as its underlying concepts and available instruments. Furthermore, we provide tables including frequency counts wherever possible. The extracted criteria were inductively clustered into newly developed main categories and subcategories. The included records were screened for concepts and terminologies of OHL to summarize how OHL in the context of OHL criteria is understood. The extracted terminologies were assigned to conceptual clusters. The publications using OHL assessment instruments were grouped by instrument.

\section{Results}

\section{Search and screening process}

A total of 639 publications were identified through a database search. After removing 322 duplicates and 16 inaccessible records, 301 articles remained for title and abstract screening during which further 179 records were excluded. After screening the remaining 122 fulltexts for eligibility, 84 more records were excluded. By screening the references of the records, 29 records were added and seven literature reviews were excluded (additional file 6 "Included and excluded records"). Finally,

${ }^{1}$ Concepts are understood as frameworks, models or definitions of OHL which were referenced to and used within the records to derive the mentioned terminologies of OHL. 
60 publications were included for data extraction and synthesis (Fig. 1).

\section{Sample of included records}

All 60 records included were published between 2006 and 2020 (Table 1).

Until 2013, most of the records were published in the USA, and in total, more than half of the records $(n=33)$ were published there. The remaining publications were conducted in six European countries (Austria, Denmark, Germany, Ireland, the Netherlands, Italy; $n=19)$, Australia $(\mathrm{n}=4)$, Canada $(\mathrm{n}=2)$, New Zealand $(\mathrm{n}=1)$, and Turkey $(\mathrm{n}=1)$. The included publications were grouped into four types: guidebook $(\mathrm{n}=10)$, journal article $(\mathrm{n}=38)$, chapter/book $(\mathrm{n}=7)$, and doctoral thesis $(\mathrm{n}=5)$. The type of study referred to the wording used in the publications or was assigned by the authors of this review. The majority was classified as toolkits $(n=15)$, discussions $(n=9)$, case studies $(n=9)$, feasibility studies $(\mathrm{n}=7)$, cross-sectional studies $(\mathrm{n}=4)$, evaluation studies $(n=4)$ or workshop summaries $(n=4)$.

\section{Synthesis}

\section{Criteria characterizing HLHCOs}

In total, 490 criteria were extracted from 60 publications (Table 2 and additional file 7 "OHL criteria by category"). In total, four criteria were assigned to two different clusters resulting in the assignment of 494 criteria including duplicates.
By looking at each criterion separately, the following six main categories of criteria were identified: 1) communication with service users, 2) easy access \& navigation, 3) integration \& prioritization of OHL, 4) assessments \& organizational development, 5) engagement \& support of service users, and 6) information \& qualification of staff. The six main categories were refined into 25 subcategories (Table 2). The number of criteria per category (last column in Table 2) shows how often they were addressed in the included publications. The frequency of a category does not necessarily equate to its importance but can illustrate an initial relationship between the criteria characterizing an HLHCO.

The most prevalent main category refers to the communication with service users, for example, patients, clients, family, or caregivers (n criteria $=190$ ). Subcategories refer to a range of aspects such as educating and informing people by "provid[ing] patient training and assistance around personal health records and health IT tools" [57] or supporting communication by "set[ting] up systems that make checking for understanding standard practice" [62]. An important aspect of educating people and health promotion is that the organization aims to reach people beyond its patients in treatment and strives to "improve HL [health literacy] in the organisation's community and catchment area" [98]. The second most represented main category is easy access $\mathcal{E}$ navigation (n criteria $=86$ ). Criteria of this cluster, for example, refer to physical access \& navigation meaning that "the organization has an easy-to-follow

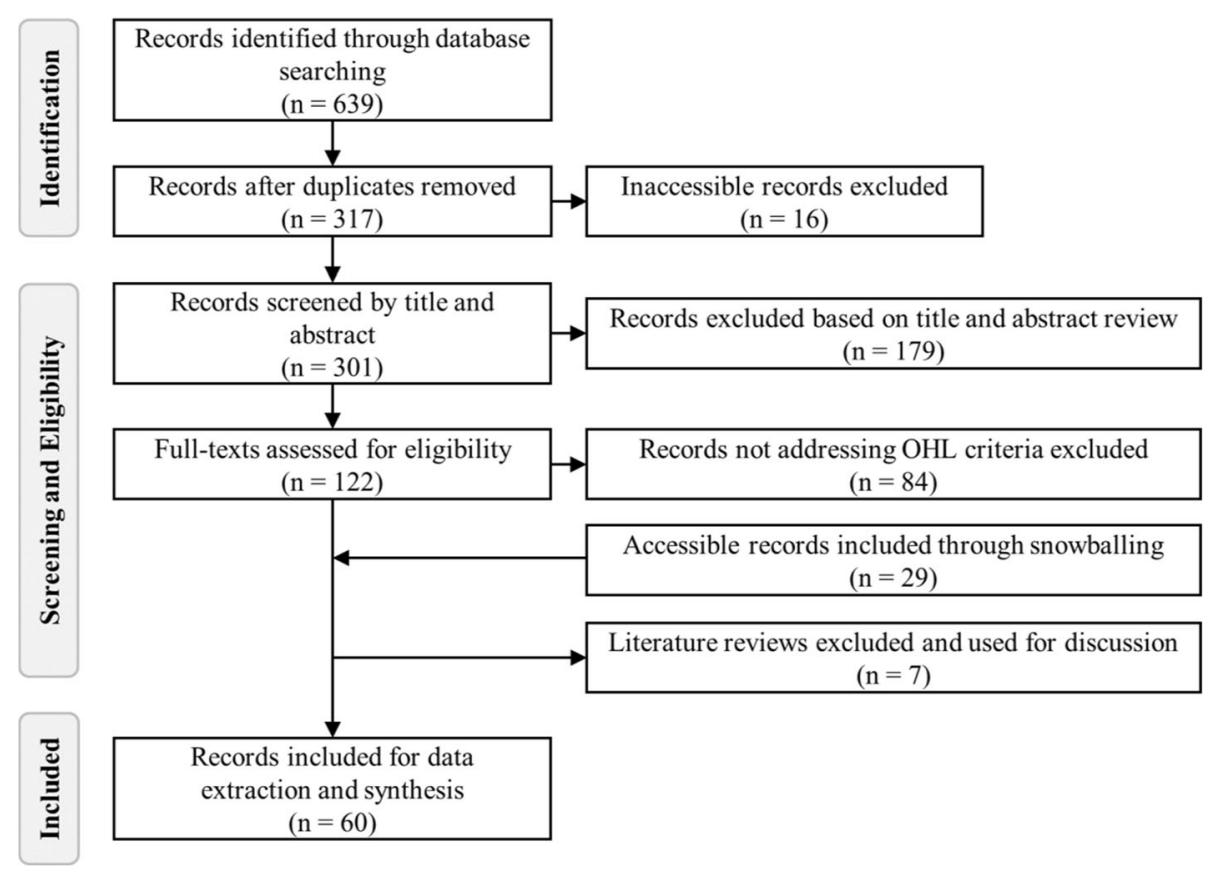

Fig. 1 Flowchart of the screening process [53] 
Table 1 Characteristics of included records

\begin{tabular}{|c|c|c|}
\hline $\begin{array}{l}\text { Author, year (sorted), } \\
\text { country }\end{array}$ & $\begin{array}{l}\text { Type of publication (type of } \\
\text { study) }\end{array}$ & Group of participants \\
\hline $\begin{array}{l}\text { Rudd \& Anderson, 2006, } \\
\text { USA [1] }\end{array}$ & Guidebook (Toolkit) & n. a. \\
\hline $\begin{array}{l}\text { Jacobson et al., 2007, USA } \\
\text { [54] }\end{array}$ & Guidebook (Toolkit) & n. a. \\
\hline NALA, 2009, Ireland [55] & Guidebook (Toolkit) & n. a. \\
\hline DeWalt et al., 2010, USA [24] & Guidebook (Toolkit) & n. a. \\
\hline Rudd, 2010, USA [56] & Guidebook (Toolkit) & n. a. \\
\hline DeWalt et al., 2011, USA [26] & $\begin{array}{l}\text { Journal article (Toolkit, feasibility } \\
\text { std.) }\end{array}$ & Staff members (eight practices) \\
\hline Brach et al., 2012, USA [2] & Journal article (Discussion) & n. a. \\
\hline $\begin{array}{l}\text { Institute of Medicine, 2012, } \\
\text { USA [57] }\end{array}$ & Chapter/book (WS) & Representatives from $\mathrm{HCO}$ \\
\hline $\begin{array}{l}\text { Parker \& Hernandez, 2012, } \\
\text { USA [58] }\end{array}$ & Journal article (WS) & Representatives from HCO \\
\hline $\begin{array}{l}\text { Six-Means et al., 2012, USA } \\
\text { [29] }\end{array}$ & $\begin{array}{l}\text { Journal article (Implementation, } \\
\text { case std.) }\end{array}$ & n. a. \\
\hline Weaver et al., 2012, USA [59] & Journal article (Case std.) & 19 staff members, 16 patients (one health center, three clinics) \\
\hline Wong, 2012, USA [52] & Journal article (Discussion) & n. a. \\
\hline $\begin{array}{l}\text { Institute of Medicine, 2013, } \\
\text { USA [60] }\end{array}$ & Chapter/book (WS) & Health literacy implementers from HCOs \\
\hline O'Neal et al., 2013, USA [61] & $\begin{array}{l}\text { Journal article (Assessment, } \\
\text { feasibility std.) }\end{array}$ & 45 staff members, 62 patients (eight pharmacies) \\
\hline $\begin{array}{l}\text { Thomacos \& Zazryn, 2013, } \\
\text { Australia [49] }\end{array}$ & Guidebook (Toolkit) & n. a. \\
\hline Abrams et al., 2014, USA [62] & Guidebook (Toolkit) & n. a. \\
\hline Brach et al., 2014, USA [23] & Journal article (Discussion) & n. a. \\
\hline Johnson, 2014, Australia [63] & Journal article (Case std.) & Two consumers (one HCO) \\
\hline $\begin{array}{l}\text { Kaphingst et al., 2014, USA } \\
\text { [64] }\end{array}$ & $\begin{array}{l}\text { Journal article (Cross-sectional } \\
\text { std.) }\end{array}$ & 3358 adults (general population) \\
\hline $\begin{array}{l}\text { Palumbo \& Annarumma, } \\
\text { 2014, Italy [65] }\end{array}$ & Journal article (Evaluation std.) & 40 healthcare professionals (various HCOs) \\
\hline Schuman, 2014, USA [22] & Chapter/book (Discussion) & n. a. \\
\hline $\begin{array}{l}\text { Altin \& Stock, 2015, } \\
\text { Germany [66] }\end{array}$ & Journal article (Discussion) & n. a. \\
\hline $\begin{array}{l}\text { Altin et al., 2015, Germany } \\
\text { [25] }\end{array}$ & Journal article (Validation std.) & 1125 adults (general population) \\
\hline Brega et al., 2015, USA [67] & Guidebook (Toolkit) & n. a. \\
\hline Briglia et al., 2015, USA [68] & Journal article (Case std.) & One health care network ( 12 health centers) \\
\hline $\begin{array}{l}\text { Kowalski et al., 2015, } \\
\text { Germany [19] }\end{array}$ & Journal article (Validation std.) & 51 directors, 1224 patients (56 hospitals) \\
\hline $\begin{array}{l}\text { Ministry of Health, 2015, } \\
\text { New Zealand [69] }\end{array}$ & Guidebook (Toolkit) & n. a. \\
\hline $\begin{array}{l}\text { Pelikan \& Dietscher, 2015, } \\
\text { Austria [70] }\end{array}$ & $\begin{array}{l}\text { Guidebook (Toolkit, feasibility } \\
\text { std.) }\end{array}$ & Three to eleven staff members per hospital (nine hospitals) \\
\hline $\begin{array}{l}\text { Pelikan \& Dietscher, 2015, } \\
\text { Austria [71] }\end{array}$ & $\begin{array}{l}\text { Journal article (Toolkit, feasibility } \\
\text { std.) }\end{array}$ & n. a. \\
\hline $\begin{array}{l}\text { Annarumma \& Palumbo, } \\
\text { 2016, Italy [72] }\end{array}$ & Journal article (Evaluation std.) & 40 healthcare professionals (various HCOs) \\
\hline $\begin{array}{l}\text { Dietscher \& Pelikan, 2016, } \\
\text { Austria [17] }\end{array}$ & $\begin{array}{l}\text { Journal article (Toolkit, feasibility } \\
\text { std.) }\end{array}$ & Three to 11 staff members per hospital (nine hospitals) \\
\hline
\end{tabular}


Table 1 Characteristics of included records (Continued)

\begin{tabular}{|c|c|c|}
\hline $\begin{array}{l}\text { Author, year (sorted), } \\
\text { country }\end{array}$ & $\begin{array}{l}\text { Type of publication (type of } \\
\text { study) }\end{array}$ & Group of participants \\
\hline Innis, 2016, Canada [73] & Doctor thesis (Pilot std.) & 42 health care experts; 99 staff members (79 hospitals) \\
\hline Napel, 2016, USA [74] & $\begin{array}{l}\text { Doctor thesis (Cross-sectional } \\
\text { std.) }\end{array}$ & 102 nurses (various hospitals) \\
\hline Adsul et al., 2017, USA [75] & Journal article (Qualitative std.) & 11 senior staff members (11 HCOs) \\
\hline Baur et al., 2017, USA [76] & Chapter/book (Discussion) & n. a. \\
\hline Brach, 2017, USA [10] & Journal article (Discussion) & n. a. \\
\hline $\begin{array}{l}\text { Dietscher \& Pelikan, 2017, } \\
\text { Austria [77] }\end{array}$ & $\begin{array}{l}\text { Chapter/book (Toolkit, feasibility } \\
\text { std.) }\end{array}$ & Three to 11 staff members per hospital (nine hospitals) \\
\hline Eigelbach, 2017, USA [78] & Journal article (Discussion) & n. a. \\
\hline $\begin{array}{l}\text { Ernstmann et al., 2017, } \\
\text { Germany [79] }\end{array}$ & Journal article (Validation std.) & Five experts; 453 patients (18 cancer centers) \\
\hline $\begin{array}{l}\text { Farmanova, 2017, Canada } \\
\text { [80] }\end{array}$ & Doctor thesis (Qualitative std.) & 12 health service org.; nine managers (eight health service org.) \\
\hline $\begin{array}{l}\text { Palumbo et al., 2017, Italy } \\
\text { [81] }\end{array}$ & Journal article (Multiple case std.) & 15 experts (three hospitals) \\
\hline Prince, 2017, USA [47] & Doctor thesis (Case std.) & 463 employees (one health center) \\
\hline $\begin{array}{l}\text { Trezona et al., 2017, Australia } \\
\text { [12] }\end{array}$ & $\begin{array}{l}\text { Journal article (Assessment, } \\
\text { evaluation std.) }\end{array}$ & 42 professionals; 153 professionals (36 health and social service org.) \\
\hline $\begin{array}{l}\text { Wieczorek et al., 2017, } \\
\text { Austria [82] }\end{array}$ & $\begin{array}{l}\text { Journal article (Assessment, } \\
\text { evaluation std.) }\end{array}$ & Six to 15 youth workers/federal state (six youth work settings in three fed. states) \\
\hline $\begin{array}{l}\text { Hayran \& Özer, 2018, Turkey } \\
\text { [83] }\end{array}$ & $\begin{array}{l}\text { Journal article (Cross-sectional } \\
\text { std.) }\end{array}$ & 18 managers and 1459 patients (three hospitals) \\
\hline $\begin{array}{l}\text { Leonard et al., 2018, USA } \\
\text { [84] }\end{array}$ & Journal article (Case std.) & One medical center \\
\hline $\begin{array}{l}\text { Oelschlegel et al., 2018, USA } \\
\text { [85] }\end{array}$ & Journal article (Case std.) & One medical center \\
\hline $\begin{array}{l}\text { Palumbo \& Annarumma, } \\
\text { 2018, Italy [86] }\end{array}$ & Journal article (Assessment std.) & 16 senior managers/staff members ( 16 pharmacies) \\
\hline Prince et al., 2018, USA [18] & Journal article (Case std.) & 463 employees (one health center) \\
\hline $\begin{array}{l}\text { Trezona et al., 2018, Australia } \\
\text { [87] }\end{array}$ & Journal article (Toolkit) & 22 health/social service professionals (22 org.) \\
\hline Trueheart, 2018, USA [50] & Doctor thesis (Multiple case std.) & 13 experts (13 health org.) \\
\hline Brega et al., 2019, USA [88] & Journal article (Delphi std.) & 18 experts ( 20 org.); one patient representative \\
\hline $\begin{array}{l}\text { Kaper et al., 2019, Ireland, } \\
\text { Netherlands [89] }\end{array}$ & $\begin{array}{l}\text { Journal article (Longitudinal } \\
\text { mixed-methods std.) }\end{array}$ & 24 staff members, 40 service users (four hospitals) \\
\hline Pelikan, 2019, Austria [90] & Chapter/book (Discussion) & n. a. \\
\hline Vamos et al., 2019, USA [51] & $\begin{array}{l}\text { Journal article (Development of } \\
\text { teaching tool) }\end{array}$ & One pediatric health clinic \\
\hline $\begin{array}{l}\text { Aaby et al., 2020, Denmark } \\
\text { [91] }\end{array}$ & $\begin{array}{l}\text { Journal article (Case std., } \\
\text { feasibility std.) }\end{array}$ & Three managers and four therapeutic teams (one rehabilitation unit) \\
\hline $\begin{array}{l}\text { Bonaccorsi et al., 2020, Italy } \\
\text { [92] }\end{array}$ & $\begin{array}{l}\text { Journal article (Cross-sectional } \\
\text { std.) }\end{array}$ & 405 healthcare managers (20 hospitals) \\
\hline $\begin{array}{l}\text { Goldsmith et al., 2020, USA } \\
\text { [93] }\end{array}$ & Journal article (Framework) & n. a. \\
\hline NASEM, 2020, USA [94] & Chapter/book (WS) & Patients; health care professionals; various stakeholders (various org.) \\
\hline $\begin{array}{l}\text { Rathmann et al., 2020, } \\
\text { Germany [48] }\end{array}$ & Journal article (Explorative std.) & $\begin{array}{l}130 \text { managers/staff ( } 130 \text { facilities for people with disabilities); eight staff members } \\
\text { (eight facilities for people with disabilities) }\end{array}$ \\
\hline
\end{tabular}

$n=60$ records; abbreviations: org. $=$ organization(s), std. $=$ study, WS = workshop summaries 
Table 2 Criteria and attributes of HLHCOs

\begin{tabular}{|c|c|}
\hline Main categories and subcategories & Number of extracted criteria \\
\hline 1. Communication with service users & 190 \\
\hline 1.1 Education \& information & 60 \\
\hline 1.2 Easy to understand written materials \& oral exchange & 45 \\
\hline 1.3 Verification of understanding \& exchange & 29 \\
\hline 1.4 HL principles of communication & 16 \\
\hline 1.5 Diversity \& awareness & 15 \\
\hline 1.6 Soft skills & 13 \\
\hline 1.7 Technology \& innovation & 12 \\
\hline 2. Easy access $\&$ navigation & 86 \\
\hline 2.1 Navigating health care services \& cooperation & 37 \\
\hline 2.2 Physical access \& navigation & 24 \\
\hline 2.3 Provision of information \& staff assistance & 10 \\
\hline 2.4 Telephone \& online navigation & 8 \\
\hline 2.5 Overall ease & 7 \\
\hline 3. Integration \& prioritization of $\mathrm{OHL}$ & 67 \\
\hline 3.1 Commitment, integration into planning & 35 \\
\hline 3.2 Dedication of resources & 21 \\
\hline 3.3 Dissemination of $\mathrm{OHL}$ & 8 \\
\hline 3.4 External factors & 3 \\
\hline 4. Assessment \& organizational development & 57 \\
\hline 4.1 Evaluation, assessment, research, quality management & 46 \\
\hline 4.2 Needs identification & 7 \\
\hline 4.3 Transformation \& development & 4 \\
\hline 5. Engagement \& support of service users & 55 \\
\hline 5.1 Consultation \& engagement of service users & 30 \\
\hline 5.2 Support for self-management & 18 \\
\hline 5.3 Family \& caregivers & 7 \\
\hline 6. Information \& qualification of staff & 39 \\
\hline 6.1 Organizational and individual health literacy of staff & 19 \\
\hline 6.2 Communication techniques & 16 \\
\hline 6.3 Professional development & 4 \\
\hline Total number of criteria (including four duplicates) & 494 \\
\hline
\end{tabular}

60 records screened $[1,2,10,12,17-19,22-26,29,47-52,54-94] ; 30$ unique sets of criteria $[1,2,12,17,19,24,29,30,48,49,55-57,59,62,65,67,69-71,76,77$, $87,88,93-98]$

navigation system and signage" [77]. Navigating the health care system involves different health care services, requiring coordination and cooperation among the health care organizations. Hence, one aim for HLHCOs should be to "make referrals easy" [67].

The main category integration $\mathcal{E}$ prioritization of $\mathrm{OHL}$ (n criteria $=67$ ) refers to the commitment of an organization, e.g. by integrating OHL into its planning and dedicating resources. An HLHCO "has leadership that makes health literacy integral to its mission, structure, and operations" [2]. External factors framing the implementation of criteria can be found in the "external policy and funding environment" [87]. HLHCOs monitor and improve their work with assessments $\mathcal{E}$ organizational development ( $\mathrm{n}$ criteria $=57$ ). They "use assessments to determine their performance and progress in promoting health literacy" [49]. The fifth main category refers to the engagement $\mathcal{E}$ support of service users ( $\mathrm{n}$ criteria $=55$ ). For example, navigation systems, services, and materials are designed by involving the peoples' perspective, "undertaking community consultation and enabling consumer participation" [87]. Finally, for implementing all these criteria the information $\mathcal{E}$ qualification of staff (n criteria $=39$ ) is pertinent for an 
HLHCO. Communication techniques are improved by "staff skills building (print communication and oral exchange)" [1] and "health promotion for staff" [48] is provided. Additional file 7 ("OHL criteria by category") shows based on which criteria the categories were formed.

\section{Underlying concepts and terminologies of OHL criteria}

The authors defined four conceptual clusters (Table 3). The first cluster, "health literacy in various social contexts" ( $\mathrm{n}=18$ records), considered the macro level and includes several societal areas, e.g. health care, education, cultural and social aspects linking them to health literacy and a system's perspective (e.g. health literacy framework). The second cluster was defined by the term "health literate health care organization" ( $\mathrm{n}=70$ records) (e.g. health literate hospitals and health centers). Organizational behavior ( $\mathrm{n}=9$ records) was the third cluster, which pooled concepts of organizational behaviors and their procedural character (e.g. organizational responsiveness, organizational learning, or transformational change). Finally, the fourth cluster "communication in health care" ( $n=6$ records) focused on the micro-level pointing out various aspects of health literacy and communication in health care (e.g. communication quality, cultural factors, or patient-centered communication).

In terms of concepts used, the majority of the included records (47 out of 60) referred to the publication "Ten Attributes of Health Literate Health Care Organizations"

Table 3 Concepts and terminologies in publications on HLHCOs

\begin{tabular}{|c|c|c|}
\hline $\begin{array}{l}\text { Concept cluster } \\
\text { Extracted terminology }\end{array}$ & $\begin{array}{l}\text { Introducing } \\
\text { reference }\end{array}$ & Referring references \\
\hline \multicolumn{3}{|c|}{ 1. Health literacy in various social contexts } \\
\hline Health literate care & [99] & {$[64,66,73,84]$} \\
\hline \multirow[t]{2}{*}{ Health literacy framework } & {$[3]$} & $\begin{array}{l}{[1,26,52,54,56,61,63,65,66,} \\
72,80]\end{array}$ \\
\hline & {$[100]$} & {$[70,77,82]$} \\
\hline
\end{tabular}

\section{Health literate health care organization}

Health literate organization (referring to health care)

Organizational health literacy (referring to health care)

Health literacy environment of hospitals and health centers; literacy-friendly healthcare facility

Literacy friendly healthcare settings

Contextualizing health literacy to health care organizations

Health literate hospitals and healthcare organizations; Organizational health literacy of

Health literate health care organization

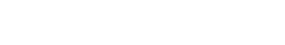
hospitals; Concept of the health literate health care organization

\section{Organizational behavior}

Health literacy universal precautions

Organizational capacity for health literacy

Organizational health literacy responsiveness

Organizational learning

Organizational model for transformational change in health care systems

\section{Communication in health care}

Communicating health

Health literacy and communication quality in health care organizations

Literacy, culture, language to improve health care quality

Patient navigation support for health literacy and patient-clinician communication

Cultural framework for health

Patient-centered communication

$\begin{array}{lll}{[33]} & {[89]} & 1 \\ {[30]} & {[88,89]} & 2 \\ {[1]} & {[2,24,26,52,54,55,63,65,66,} & 11 \\ & 69,84] & 1 \\ {[55]} & {[55]} & 1 \\ {[72]} & {[89]} & 2 \\ {[71,72,77,98]} & {[71,77,89,90,98]} & \\ & & \\ {[2]} & {[10,12,17-19,22,23,25,29,47-} & 47 \\ & 52,58-60,62-66,68-80,82-90, & \\ {[57]} & 92,93] & 5\end{array}$

18 *

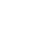


by Brach et al. [2]. Accordingly, an HLHCO is defined as "an organisation that makes it easier for people to navigate, understand, and use information and services to take care of their health" [2]. 11 records (see Table 3 "Health literacy framework") related to the work of the Institute of Medicine by stating that "health literacy goes beyond the individual obtaining information. Health literacy emerges when the expectations, preferences, and skills of individuals seeking health information and services meet the expectations, preferences, and skills of those providing information and services. Health literacy arises from a convergence of education, health services, and social and cultural factors" [3]. The publication "The Health Literacy Environment of Hospitals and Health Centers. Partners for Action: Making Your Healthcare Facility Literacy-Friendly" by Rudd \& Anderson [1] was the conceptual basis of 11 records. The remaining publications were extracted five times or less.

\section{Instruments to measure $\mathrm{OHL}$ criteria}

In total, 17 assessment tools and instruments were extracted from 36 records (Table 4). The "Health Literate Health Care Organization 10 Item Questionnaire (HLHO-10)" [19], "Health Literacy Universal Precautions Toolkit" [24, 67], and the "Health Literacy Environment of Hospitals and Health Centers (HLEHHC)" [1] were referenced the most within our sample of 60 records. Only some of the toolkits and instruments were validated or tested in feasibility studies $[25,26,61,70$, $79,91]$. The methods of assessment were multifaceted, e.g. standardized questionnaires, semi-structured interviews, observations, checklists, and material assessments. Mainly, the existing tools were applied as surveys via questionnaires. The tools were either used in their original form (e.g. [19]) or by selecting a subset of items or tools (e.g. [86] using the Pharmacy Health Literacy Assessment Tool [54]), translating the tool into a different language [83], extending and adapting the contents [73] or adapting the measures for a different type of method [64].

\section{Discussion}

\section{Summary of findings}

This scoping review provides a comprehensive overview of OHL of health care organizations by synthesizing the current status of publications on criteria that characterize HLHCOs. Furthermore, we gathered information on which understanding of OHL these criteria are based on and how they are measured.

Our review included 60 publications on OHL criteria. We extracted 490 criteria (494 with duplicates) characterizing HLHCOs which were clustered into six main categories (Table 2). Rowlands et al. [31] stated a similar number and nature of categories in a literature review including some frameworks. In their recent review, Zanobini et al. [20] focused on hospitals, their characteristics, and interventions regarding OHL, and found that most of the included studies focused on contents easy to understand.

Table 4 Instruments to measure the health literacy of health care organizations

\begin{tabular}{|c|c|}
\hline Assessment tools \& instruments & Applied by records \\
\hline 1. Communication Climate Assessment Toolkit (C-CAT) [104] & {$[65,72]$} \\
\hline 2. Consumer Assessment of Health Providers and Systems (CAHPS ${ }^{\circledR}$ Clinician \& Group Survey) [109] & [64] \\
\hline 3. Enliven Organisational Health Literacy Self-assessment [49] & [49] \\
\hline 4. Health Literacy Environment of Hospitals and Health Centers (HLEHHC) [1] & {$[1,59,81,84,85]$} \\
\hline 5. Health Literacy Review [69] & [69] \\
\hline 6. Health Literacy Universal Precautions Toolkit $[24,67]$ & {$[24,26,59,67,74,80]$} \\
\hline 7. Health Literacy-Sensitivity of Communication (HL-COM) [79] & [79] \\
\hline 8. Health Literate Discharge Practices [96] & [73] \\
\hline 9. Health Literate Health Care Organization 10 Item Questionnaire (HLHO-10) [19] & {$[18,19,47,48,80,83,92]$} \\
\hline 10. Health Literate Primary Care Practice Screener (HLPC) [25] & {$[25]$} \\
\hline 11. Literacy Audit for Health Care Settings [55] & {$[55,89]$} \\
\hline 12. Organisational Health Literacy Responsiveness (Org-HLR) self-assessment tool and process [87] & {$[87,91]$} \\
\hline 13. Patient Education Materials Assessment Tool (PEMAT) [110] & {$[84]$} \\
\hline 14. Pharmacy Health Literacy Assessment Tool [54] & {$[54,61,86]$} \\
\hline 15. Quickscan Health Literacy Toolbox [111] & {$[89]$} \\
\hline 16. The Health Literacy Environment Activity Packet [56] & {$[56,63]$} \\
\hline 17. Vienna Concept of Health-Literate Hospitals and Healthcare Organizations (V-HLO-I) [70] & {$[17,70,77]$} \\
\hline
\end{tabular}


In line with this assessment, the largest main category of criteria identified by our review was communication with service users. More than half of the criteria assigned to this category were clustered into education $\mathcal{E}$ information and easy to understand written materials $\mathcal{E}$ oral exchange. While materials for patients are one of the main areas of work for OHL, we found a variety of other aspects relevant to the implementation of OHL criteria. Next to physical access to health care facilities, examples range from the participation and commitment of consumers to the organization's commitment to OHL to a degree that OHL is widely integrated into its planning. An organization's efforts to advocate for health literacy go beyond treating and educating its users. Instead, an HLHCO is also committed to promoting the health of its staff $[2,17,48,77,95,98]$ and the regional community $[17,76,77,98]$.

By investigating OHL guides, Farmanova et al. [30] showed that all reviewed guides included the items communication and access and navigation and that the workforce plays an important role not only in health care but also in the creation of a health literate environment. Furthermore, leadership was crucial by integrating health literacy in an organization's vision, mission, and strategic planning [30].

Our review underlines the large number of OHLrelated criteria indicating that OHL is a complex construct potentially including a significant number of organizational criteria that specify and expand the "Ten attributes of health literate health care organizations" [2, 12, 67, 90]. Consequently, becoming a health literate health care organization requires a holistic approach [90].

In our review, 60 records were screened for concepts and terminologies related to health literacy and organizational contexts (Table 3). The terminologies were extracted and assigned to four conceptual clusters. The concepts of Brach et al. [2], Institute of Medicine [3], and Rudd \& Anderson [1] were the dominating ones in this context.

Meggetto et al. [34] identified 19 different terms to describe OHL, the three most frequent terms being health system health literacy, organizational health literacy, and health literacy practice/s. They observed that different terms overlap in content, but aim at different levels or contexts and concluded an interdependence between the terms and concepts: "Within health system health literacy sits OHL, and within this sits health literacy practice" [34].

Our results substantiated that a broad range of OHL terminologies based on various concepts exist in the OHL literature. This versatility represents the multi-level approach that is needed to incorporate the concept of OHL. An integrative framework of OHL could include micro- (e.g. individual behavior), meso- (e.g. organizational processes), and macro-level (e.g. health policies) aspects [90].

Our review shows that 17 different assessment tools and instruments were used (Table 4). The methods of assessment were versatile, but most of them used surveys via questionnaires. In their review, Kripalani et al. systematically described existing measures and found that "most measures have been developed with strong content validity, but little research has been done to examine their internal reliability, construct, or predictive validity" [35]. Rowlands et al. also "found limited evidence of the use of organizational health literacy/responsiveness measures and tools as part of an evaluation of a programme or intervention" [31]. Lloyd et al. stated in their study, searching for effective strategies for creating $\mathrm{OHL}$, that "the use of health literacy tools proved important for raising awareness of health literacy issues within organisations, these tools were insufficient for generating the organisational changes necessary to improve organisational health literacy" [33].

Our review confirmed that a large number of toolkits and instruments to measure and assess OHL was published. Most of them were developed inductively, but with a practical orientation by including health care experts and service users $[12,87]$. Only a few of them were validated or showed proof of feasibility $[19,77,91]$.

To prevent overloading this manuscript, not all results are shown in detail. Following our study protocol, we also analyzed data on further involved persons and stakeholders (additional file 3), organizational processes (additional file 2) and implemented interventions to become an HLHCO (additional file 4). Our analyses showed that the involvement of service users either as part of a (potential) target group for an OHL instrument or in an active role during the implementation process itself still happens too rarely. More interventions and evaluations regarding activities of organizational change on OHL, such as the preparation and assessment stage of processes, are needed. Health service research should be included and consulted to support and monitor processes to become an HLHCO.

\section{Limitations}

Literature reviews have to deal with the risk of publication bias. We were able to minimize it by using seven databases, snowballing techniques, and no restrictions regarding the source of information. The scoping review captured different types of publications (e.g. guidebooks, journal articles, doctoral thesis, and book chapters) to give a comprehensive overview of the subject. Nevertheless, some relevant records may have been omitted due to the absence of pertinent keywords in the search strings. Due to the characteristics of a scoping review, 
the quality of the publications was not evaluated. Notably, some of the presented toolkits and instruments were validated or tested in feasibility studies [25, 26, 61, $70,79,91]$. Furthermore, the findings may be limited to health care organizations in Western countries, since more than two-thirds of the records (42 out of 60) were from North America (USA = 33, Canada =2) and other English-speaking countries (Australia $=4$, Ireland $=2$, New Zealand =1). Lastly, only English- and Germanlanguage publications were taken into account due to time and cost considerations.

\section{Conclusions}

The idea of OHL turned the tables. It is not the patients and other service users who need to adapt, it is the health care organization's responsibility to make "it easy for people to navigate, understand, and use information and services to take care of their health" [10]. This study showed which criteria of health care organizations make an organization health literate, which underlying concepts or terminologies exist, and what instruments are used to measure OHL criteria.

Since OHL is a complex construct, future research should focus on providing evidence on effective and efficient core elements of OHL to support the implementation of OHL criteria into health care practice. Furthermore, a consensus-based framework of organizational health literacy that is theoretically sound is still needed. Health services research should provide validated tools and instruments which are sensitive, but also feasible and easy for health care organizations to integrate into their daily routine. Before starting their "journey to become a health literate organization" [10] health care organizations should carefully define theirs aims, assess theirs needs and plan their resources. Considering that, the large number of OHL-related criteria may imply the necessity to focus on just a few aspects of OHL in the beginning.

\section{Abbreviations \\ AHRQ: Agency for Healthcare Research and Quality; HL: Health literacy; HLHCO: Health literate health care organization; IOM: Institute of Medicine; NALA: National Adult Literacy Agency; NAM: The National Academy of Medicine (formerly IOM); OHL: Organizational health literacy; OHL- HAM: Research project on OHL in the Hamburg Metropolitan Area (Germany); PICo: Population, Interest, Context; PRISMA-ScR: Preferred Reporting Items for Systematic reviews and Meta-Analyses extension for Scoping Reviews Checklist}

\section{Supplementary Information}

The online version contains supplementary material available at https://doi. org/10.1186/s12913-021-06604-z

Additional file 1. PRISMA-SCR Checklist. Filled PRISMA-SCR checklist. Additional file 2. Process stages of becoming an HLHCO. Synthesized process stages and examples for becoming an HLHCO.
Additional file 3. Involved actors by type of organization. Actors involved in the process of becoming an HLHCO by type of organization.

Additional file 4. Implemented interventions. Implemented interventions to promote $\mathrm{OHL}$ grouped by category of criteria.

Additional file 5. Search details. Search string used for PubMed and search settings used for CINAHL, Cochrane Library, JSTOR, PsycINFO, PubMed, Web of Science Core Collection, Wiley Online Libary.

Additional file 6. Included and excluded records. List of included and excluded records based on full-text screening (including records identified through snowballing).

Additional file 7. OHL criteria by category. Detailed list of extracted $\mathrm{OHL}$ criteria and records sorted by main and subcategory.

\section{Acknowledgments}

None.

\section{Authors' contributions}

$\mathbb{I K}, \mathrm{DL}$, and $\mathrm{DB}$ designed the study. IK, $\mathrm{L}, \mathrm{DL}$ and $\mathrm{DB}$ collected and validated the data. IK and DB analyzed and visualized the data. DB and IK wrote the original draft. $\mathrm{DL}, \mathrm{LJ}, \mathrm{OK}$, and $\mathrm{MH}$ reviewed and edited the manuscript. All authors read and approved the manuscript.

\section{Funding}

This research is part of the research project OHL-HAM (Health Literate Health Care Organizations in the Region of Hamburg) funded by the German Federal Ministry of Education and Research (01GY1912). Open Access funding enabled and organized by Projekt DEAL.

\section{Availability of data and materials}

The search syntax, checklist, list of extractions, and included and excluded studies are provided as additional files. Please see supplementary materials.

\section{Declarations}

Ethics approval and consent to participate

Due to the nature of a scoping review, neither ethics approval nor consent to participate was required.

\section{Consent for publication}

Not applicable.

\section{Competing interests}

The authors declare that they have no competing interests.

\section{Author details}

${ }^{1}$ Department of Medical Psychology, Center for Health Care Research, University Medical Center Hamburg-Eppendorf, Martinistr. 52, 20246 Hamburg, Germany. ${ }^{2}$ Department of Medical Sociology, Center for Health Care Research, University Medical Center Hamburg-Eppendorf, Martinistr. 52, 20246 Hamburg, Germany.

Received: 17 March 2021 Accepted: 2 June 2021

Published online: 06 July 2021

\section{References}

1. Rudd RE, Anderson JE. The health literacy environment of hospitals and health centers. Boston: MA; 2006

2. Brach C, Keller D, Hernandez LM, Baur C, et al. Ten Attributes of Health Literate Health Care Organizations. 2012. https://nam.edu/wp-content/uploa ds/2015/06/BPH_Ten_HLit_Attributes.pdf. Accessed 15 Sept 2020.

3. Institute of Medicine. Health literacy: a prescription to end confusion. Washington, DC: National Academies Press; 2004.

4. Bröder J, Chang P, Kickbusch I, Levin-Zamir D, McElhinney E, Nutbeam D, et al. IUHPE position statement on health literacy: a practical vision for a health literate world. Glob Health Promot. 2018;25(4):79-88 https://doi.org/10.11 77/1757975918814421

5. Sørensen K, van den Broucke S, Fullam J, Doyle G, et al. Health literacy and public health: a systematic review and integration of definitions and 
models. BMC Public Health. 2012;12(1):80 https://doi.org/10.1186/1471-24 58-12-80.

6. Schaeffer D, Berens E-M, Gille S, Griese L, et al. Gesundheitskompetenz der Bevölkerung in Deutschland vor und während der Corona Pandemie: Ergebnisse des HLS-GER 2. Bielefeld: Universität Bielefeld, Interdisziplinäres Zentrum für Gesundheitskompetenzforschung (IZKG); 2021.

7. Sørensen K, Pelikan JM, Röthlin F, Ganahl K, Slonska Z, Doyle G, et al. Health literacy in Europe: comparative results of the European health literacy survey (HLS-EU). Eur J Pub Health. 2015;25(6):1053-8 https://doi.org/10.1093/ eurpub/ckv043.

8. Hurrelmann K, Klinger J, Schaeffer D. Gesundheitskompetenz der Bevölkerung in Deutschland: Vergleich der Erhebungen 2014 und 2020. Bielefeld: Universität Bielefeld, Interdisziplinäres Zentrum für Gesundheitskompetenzforschung (IZKG); 2020

9. Kickbusch I, Pelikan JM, Apfel F, Tsouro AD. Health literacy. The solid facts. Geneva: World Health Organization; 2013.

10. Brach C. The journey to become a health literate organization: a snapshot of health system improvement. Stud Health Technol Inform. 2017;240:20337 https://doi.org/10.3233/978-1-61499-790-0-203.

11. Berkman ND, Sheridan SL, Donahue KE, Halpern DJ, Crotty K. Low health literacy and health outcomes: an updated systematic review. Ann Intern Med. 2011;155(2):97-107 https://doi.org/10.7326/0003-4819-155-2-2011071 90-00005.

12. Trezona A, Dodson S, Osborne RH. Development of the organisational health literacy responsiveness (org-HLR) framework in collaboration with health and social services professionals. BMC Health Serv Res. 2017;17(1):112 https://doi.org/10.1186/s12913-017-2465-z.

13. Palumbo R. Examining the impacts of health literacy on healthcare costs. An evidence synthesis. Health Serv Manag Res. 2017;30(4):197-212 https://doi. org/10.1177/0951484817733366.

14. Penaranda E, Diaz M, Noriega O, Shokar N. Evaluation of health literacy among Spanish-speaking primary care patients along the US-Mexico border. South Med J. 2012;105(7):334-8 https://doi.org/10.1097/SMJ.0b013e3182 $5 b 2468$.

15. Paasche-Orlow M. Caring for patients with limited health literacy: a 76-yearold man with multiple medical problems. JAMA. 2011:306(10):1122-9 https://doi.org/10.1001/jama.2011.1203.

16. Parker RM, Wolf MS, Kirsch I. Preparing for an epidemic of limited health literacy: weathering the perfect storm. J Gen Intern Med. 2008;23(8):1273-6 https://doi.org/10.1007/s11606-008-0621-1.

17. Dietscher C, Pelikan JM. Gesundheitskompetente Krankenbehandlungsorganisationen. Pravention Und Gesundheitsforderung. 2016;11(1):53-62 https://doi.org/10.1007/s11553-015-0523-0.

18. Prince LY, Schmidtke C, Beck JK, Hadden KB. An assessment of organizational health literacy practices at an academic health center. Qual Manag Health Care. 2018;27(2):93-7 https://doi.org/10.1097/QMH. 0000000000000162

19. Kowalski C, Lee S-YD, Schmidt A, Wesselmann S, Wirtz MA, Pfaff H, et al. The health literate health care organization 10 item questionnaire (HLHO-10): development and validation. BMC Health Serv Res. 2015;15(1):47 https://doi. org/10.1186/s12913-015-0707-5.

20. Zanobini P, Lorini C, Baldasseroni A, Dellisanti C, Bonaccorsi G. A scoping review on how to make hospitals health literate healthcare organizations. Int J Environ Res Public Health. 2020;17(3) https://doi.org/10.3390/ijerph1 7031036.

21. Henrard G, Vanmeerbeek M, Dardenne N, Rademakers J. The Vienna selfassessment questionnaire: a usable tool towards more health-literate hospitals? Explorative case studies in three hospitals in Belgium. BMC Health Serv Res. 2021;21(1):287 https://doi.org/10.1186/s12913-021-06211-y.

22. Schuman MJ. Policy implications for advanced practice registered nurses: quality and safety. In: Goudreau KA, Smolenski MC, editors. Health policy and advanced practice nursing: impact and implications. New York: Springer Publishing Company LLC; 2014. p. 253-71.

23. Brach C, Dreyer BP, Schillinger D. Physicians' roles in creating health literate organizations: a call to action. J Gen Intern Med. 2014;29(2):273-5 https:// doi.org/10.1007/s11606-013-2619-6.

24. DeWalt DA, Callahan LF, Hawk VH, Broucksou KA, et al. Health Literacy Universal Precautions Toolkit. Rockville: Agency for Healthcare Research and Quality; 2010.

25. Altin SV, Lorrek K, Stock S. Development and validation of a brief screener to measure the health literacy responsiveness of primary care practices
(HLPC). BMC Fam Pract. 2015;16(1):1-8 https://doi.org/10.1186/s12875-0150336-4.

26. DeWalt DA, Broucksou KA, Hawk VH, Brach C, et al. Developing and testing the health literacy universal precautions toolkit. Nurs Outlook. 2011;59(2):8594 https://doi.org/10.1016/j.outlook.2010.12.002.

27. de Gani SM, Nowak-Flück D, Nicca D, Vogt D. Self-assessment tool to promote organizational health literacy in primary care settings in Switzerland. Int J Environ Res Public Health. 2020;17(24) https://doi.org/10.33 90/ijerph17249497.

28. Salem J, Paffenholz P, Bolenz C, von Brandenstein M, Cebulla A, Haferkamp A, et al. Websites on bladder Cancer: an appropriate source of patient information? J Cancer Educ. 2019;34(2):381-7 https://doi.org/10.1007/s131 87-017-1316-2.

29. Six-Means A, Bauer TK, Teeter R, Segraves D, Cutshaw L, High L. Building a Foundation of Health Literacy with ask me $3^{\text {Tm }}$. J Consumer Health Internet. 2012;16(2):180-91 https://doi.org/10.1080/15398285.2012.673461.

30. Farmanova E, Bonneville L, Bouchard L. Organizational Health Literacy: Review of Theories, Frameworks, Guides, and Implementation. Issues. 2018; 55:46958018757848 https://doi.org/10.1177/0046958018757848.

31. Rowlands G, Trezona A, Russell S, Lopatina M, et al. What is the evidence on the methods, frameworks and indicators used to evaluate health literacy policies, programmes and interventions at the regional, national and organizational levels? Copenhagen: WHO Regional Office for Europe; 2019.

32. Palumbo R. Designing health-literate health care organization: a literature review. Health Serv Manag Res. 2016;29(3):79-87 https://doi.org/10.1177/ 0951484816639741.

33. Lloyd JE, Song HJ, Dennis SM, Dunbar N, Harris E, Harris MF. A paucity of strategies for developing health literate organisations: a systematic review. PLoS One. 2018;13(4):e0195018 https://doi.org/10.1371/journal.pone.019501 8.

34. Meggetto E, Kent F, Ward B, Keleher H. Factors influencing implementation of organizational health literacy: a realist review. J Health Organ Manag. 2020;34(4):385-407 https://doi.org/10.1108/JHOM-06-2019-0167.

35. Kripalani S, Wallston K, Cavanaugh KL, Osborn CY, et al. Measures to assess a health-literate organization. Nashville: Vanderbilt Center for Effective Health Communication; 2014

36. Lüdecke $D$, Härter M, Bremer D, Klockmann I, von dem Knesebeck O. Organisational health literacy in the Hamburg metropolitan area: the OHLHAM study. 2021. https://osf.io/mnt3f. Accessed 1 Mar 2021.

37. Munn Z, Peters MDJ, Stern C, Tufanaru C, McArthur A, Aromataris E. Systematic review or scoping review? Guidance for authors when choosing between a systematic or scoping review approach. BMC Med Res Methodol. 2018;18(1):143 https://doi.org/10.1186/s12874-018-0611-x.

38. Arksey H, O'Malley L. Scoping studies: towards a methodological framework. Int J Soc Res Methodol. 2005;8(1):19-32 https://doi.org/10.1080/1364557032 000119616.

39. Peters M, Godfrey C, Mclnerney P, Munn Z, et al. Chapter 11: Scoping Reviews. In: Aromataris E, Munn Z, editors. JBI Manual for Evidence Synthesis: JBl; 2020. https://doi.org/10.46658/JBIMES-20-12.

40. Peters MDJ, Marnie C, Tricco AC, Pollock D, et al. Updated methodological guidance for the conduct of scoping reviews. JBI Evid Synth. 2020;18:211926 https://doi.org/10.11124/JBIES-20-00167.

41. Tricco AC, Lillie E, Zarin W, O'Brien KK, Colquhoun H, Levac D, et al. PRISMA extension for scoping reviews (PRISMA-ScR): checklist and explanation. Ann Intern Med. 2018;169(7):467-73 https://doi.org/10.7326/M18-0850.

42. Campbell M, McKenzie JE, Sowden A, Katikireddi SV, et al. Synthesis without meta-analysis (SWiM) in systematic reviews: reporting guideline. BMJ. 2020; 368:16890 https://doi.org/10.1136/bmj.16890.

43. Klockmann I, Lüdecke D, Härter M, von dem Knesebeck O, Bremer D. Which criteria characterize a health literate healthcare organization? - a scoping review protocol on organizational health literacy. 2021. https:/osf.io/w9tup. Accessed 9 Feb 2021.

44. Murdoch University. Systematic reviews - research guide. 2020. https:// libguides.murdoch.edu.au/systematic/PICO. Accessed 7 July 2020.

45. Armstrong R, Hall BJ, Doyle J, Waters E. Cochrane update. 'Scoping the scope' of a cochrane review. J Public Health. 2011;33(1):147-50 https://doi. org/10.1093/pubmed/fdr015

46. Valaitis R, Martin-Misener R, Wong ST, MacDonald M, Meagher-Stewart D, Austin P, et al. Methods, strategies and technologies used to conduct a scoping literature review of collaboration between primary care and public 
health. Prim Health Care Res Dev. 2012;13(03):219-36 https://doi.org/10.101 7/S1463423611000594.

47. Prince LY. Assessing Organizational Health Literacy at an academic health center: A quantitative research study. Fayetteville: ProQuest Information \& Learning; US; 2017.

48. Rathmann K, Vockert T, Wetzel LD, Lutz J, Dadaczynski K. Organizational health literacy in facilities for people with disabilities: first results of an explorative qualitative and quantitative study. Int J Environ Res Public Health. 2020;17(8) https://doi.org/10.3390/ijerph17082886.

49. Thomacos N, Zazryn T. Enliven Organisational Health Literacy Selfassessment Resource. Melbourne: Enliven \& School of Primary Health Care, Monash University; 2013.

50. Trueheart SL. Health literacy best practices in policy development. US: ProQuest Information \& Learning; US. 2018.

51. Vamos CA, Thompson EL, Griner SB, Liggett LG, Daley EM. Applying organizational health literacy to maternal and child health. Matern Child Health J. 2019;23(5):597-602 https://doi.org/10.1007/s10995-018-2687-7.

52. Wong BK. Building a health literate workplace. Workplace Health Saf. 2012; 60(8):363-9 https://doi.org/10.3928/21650799-20120726-67.

53. Moher D, Liberati A, Tetzlaff J, Altman DG. Preferred reporting items for systematic reviews and meta-analyses: the PRISMA statement. PLoS Med. 2009;6(7):e1000097 https://doi.org/10.1371/journal.pmed.1000097.

54. Jacobson KL, Gazmararian JA, Kripalani S, McMorris KJ, et al. Is our pharmacy meeting patients' needs? Rockville: A Pharmacy Health Literacy Assessment Tool User's Guide; 2007.

55. NALA. Literacy audit for healthcare settings. Dublin: NALA; 2009.

56. Rudd RE. The health literacy environment activity packet: first Impressions \& Walking Interview. Cambridge: Harvard University; 2010.

57. Institute of Medicine. How Can Health Care Organizations Become More Health Literate?: Workshop Summary. Washington, DC: National Academies Press; 2012

58. Parker RM, Hernandez LM. What makes an organization health literate? J Health Commun. 2012;17(5):624-7 https://doi.org/10.1080/10810730.2012. 685806.

59. Weaver NL, Wray RJ, Zellin S, Gautam K, Jupka K. Advancing organizational health literacy in health care organizations serving high-needs populations: a case study. J Health Commun. 2012;17(sup3):55-66 https://doi.org/10.1 080/10810730.2012.714442

60. Institute of Medicine. Organizational Change to Improve Health Literacy: Workshop Summary. Washington, DC: National Academies Press; 2013.

61. O'Neal KS, Crosby KM, Miller MJ, Murray KA, Condren ME. Assessing health literacy practices in a community pharmacy environment: experiences using the AHRQ pharmacy health literacy assessment tool. Res Soc Admin Pharm. 2013;9(5):564-96 https://doi.org/10.1016/j.sapharm.2012.09.005.

62. Abrams MA, Kurtz-Rossi S, Riffenburgh A, Savage B. Building Health Literate Organizations: A Guidebook to Achieving Organizational Change. 2014. http://www.HealthLiterateOrganization.org. Accessed 15 Sept 2020.

63. Johnson A. First impressions: towards becoming a health-literate health service. Aust Health Rev. 2014;38(2):190-3 https://doi.org/10.1071/AH13194.

64. Kaphingst KA, Weaver NL, Wray RJ, Brown MLR, Buskirk T, Kreuter MW. Effects of patient health literacy, patient engagement and a system-level health literacy attribute on patient-reported outcomes: a representative statewide survey. BMC Health Serv Res. 2014;14(1):475 https://doi.org/10.11 86/1472-6963-14-475.

65. Palumbo R, Annarumma C. The Importance of Being Health Literate: An Organizational Health Literacy Approach. Liverpool: Liverpool John Moores University; 2014

66. Altin SV, Stock S. Health literate healthcare organizations and their role in future healthcare. Journal of Nursing \& Care. 2015;04(02) https://doi.org/1 0.4172/2167-1168.1000238.

67. Brega AG, Barnard J, Mabachi NM, Weiss BD, et al. AHRQ health literacy universal precautions toolkit. 2nd ed. Rockville: Agency for Healthcare Research and Quality; 2015.

68. Briglia E, Perlman M, Weissman MA. Integrating health literacy into organizational structure. Physician Leadersh J. 2015;2(2):66-9.

69. Ministry of Health. Health Literacy Review: A Guide. Wellington: Ministry of Health; 2015.

70. Pelikan JM, Dietscher C. Die Gesundheitskompetenz von Gesundheitseinrichtungen entwickeln: Strategien und Beispiele. Wien: Verein ONGKG; 2015.
71. Pelikan JM, Dietscher C. Why should and how can hospitals improve their organizational health literacy? Bundesgesundheitsblatt Gesundheitsforschung Gesundheitsschutz. 2015;58(9):989-95 https://doi. org/10.1007/s00103-015-2206-6.

72. Annarumma C, Palumbo R. Contextualizing health literacy to health care organizations. J Health Manag. 2016;18(4):611-24 https://doi.org/10.1177/ 0972063416666348.

73. Innis JA. Health literate discharge practices in Ontario hospitals. US: ProQuest Information \& Learning; US. 2016.

74. Napel AT. Nurses' perceptions of importance and achievability of the ten attributes of health literate healthcare organizations in their institutions: a descriptive study. 2016. https://digitalcommons.molloy.edu/etd/34. Accessed 25 Feb 2021.

75. Adsul P, Wray RJ, Gautam K, Jupka K, Weaver N, Wilson K. Becoming a health literate organization: formative research results from healthcare organizations providing care for undeserved communities. Health Serv Manag Res. 2017;30(4):188-96 https://doi.org/10.1177/0951484817727130.

76. Baur C, Harris LM, Squire E. The U.S. National Action Plan to improve health literacy: a model for positive organizational change. Stud Health Technol Inform. 2017;240:186-202 https://doi.org/10.3233/978-1-61499-790-0-186.

77. Dietscher C, Pelikan JM. Health-literate hospitals and healthcare organizations-results from an Austrian feasibility study on the selfassessment of organizational health literacy in hospitals. In: Schaeffer D, Pelikan JM, editors. Health literacy: Forschungsstand und Perspektiven. 1st ed. Bern: Hogrefe; 2017. p. 303-14.

78. Eigelbach B. Ten Suggested health literacy attributes of a health care organization. J Consumer Health Internet. 2017;21 (2):201-8 https://doi.org/1 $0.1080 / 15398285.2017 .1311606$

79. Ernstmann N, Halbach S, Kowalski C, Pfaff H, Ansmann L. Measuring attributes of health literate health care organizations from the patients' perspective: development and validation of a questionnaire to assess health literacy-sensitive communication (HL-COM). Z Evid Fortbild Qual Gesundhwes. 2017;121:58-63 https://doi.org/10.1016/j.zefq.2016.12.008.

80. Farmanova E. Organization of health services for minority populations: the role of organizational health literacy and an active offer of health services in French in Ontario. Ottawa: uO Research; 2017.

81. Palumbo R, Annarumma C, Musella M. Exploring the meaningfulness of healthcare organizations: a multiple case study. Int J Public Sect Manag. 2017;30(5):503-18 https://doi.org/10.1108/JPSM-10-2016-0174.

82. Wieczorek CC, Ganahl K, Dietscher C. Improving organizational health literacy in extracurricular youth work settings. Health Lit Res Pract. 2017;1(4): e233-8 https://doi.org/10.3928/24748307-20171101-01.

83. Hayran O, Özer O. Organizational health literacy as a determinant of patient satisfaction. Public Health. 2018;163:20-6 https://doi.org/10.1016/j.puhe.2018. 06.011 .

84. Leonard K, Oelschlegel S, Tester E, Russomanno J, Heidel RE. Assessing the print communication and technology attributes of an Academic Medical Center. Health Lit Res Pract. 2018;2(1):e26-34 https://doi.org/10.3928/247483 07-20180108-01.

85. Oelschlegel S, Grabeel KL, Tester E, Heidel RE, Russomanno J. Librarians promoting changes in the health care delivery system through systematic assessment. Med Ref Serv Q. 2018;37(2):142-52 https://doi.org/10.1080/02 763869.2018.1439216.

86. Palumbo R, Annarumma C. Empowering organizations to empower patients: an organizational health literacy approach. Int J Healthcare Manage. 2018;11(2):133-42 https://doi.org/10.1080/20479700.2016.1253254.

87. Trezona A, Dodson S, Osborne RH. Development of the Organisational Health Literacy Responsiveness (Org-HLR) self-assessment tool and process. BMC Health Serv Res. 2018;18:N.PAG-N.PAG https://doi.org/10.1186/s12913018-3499-6.

88. Brega AG, Hamer MK, Albright K, Brach C, Saliba D, Abbey D, et al. Organizational health literacy: quality improvement measures with expert consensus. Health Lit Res Pract. 2019;3(2):e127-46 https://doi.org/10.3928/24 748307-20190503-01.

89. Kaper M, Sixsmith J, Meijering L, Vervoordeldonk J, Doyle P, Barry MM, et al. Implementation and long-term outcomes of Organisational health literacy interventions in Ireland and the Netherlands: a longitudinal mixed-methods study. Int J Environ Res Public Health. 2019;16(23) https://doi.org/10.3390/ ijerph16234812.

90. Pelikan JM. Health-literate health care organisations. In: Okan O, Bauer U, Levin-Zamir D, Pinheiro P, Sørensen K, editors. International handbook of 
health literacy: Research, practice and policy across the life-span. Bristol: Policy Press; 2019. p. 539-53. https://doi.org/10.5993/AJHB.31.s1.16.

91. Aaby A, Palner S, Maindal HT. Fit for diversity: a staff-driven organizational development process based on the organizational health literacy responsiveness framework. Health Lit Res Pract. 2020;4(1):e79-83 https://doi. org/10.3928/24748307-20200129-01.

92. Bonaccorsi G, Romiti A, lerardi F, Innocenti M, del Riccio M, Frandi S, et al. Health-literate healthcare organizations and quality of Care in Hospitals: a cross-sectional study conducted in Tuscany. Int J Environ Res Public Health. 2020;17(7) https://doi.org/10.3390/ijerph17072508.

93. Goldsmith JV, Wittenberg E, Parnell TA. The COMFORT communication model: a nursing resource to advance health literacy in organizations. J Hospice Palliative Nurs. 2020;22(3):229-37 https://doi.org/10.1097/NJH. 0000000000000647.

94. National Academies of Sciences, Engineering, and Medicine. Health Literacy and Communication Strategies in Oncology: Proceedings of a Workshop. Washington, DC: National Academies Press; 2020

95. Bundesweites Netzwerk Offene Jugendarbeit (bOJA) [Centre of Competence for Open Youth Work in Austria], Bundesnetzwerk Österreichische Jugendinfos (bÖJl) (Austrian Youth Information Center). Leitfaden: Die gesundheitskompetente Offene Jugendarbeit \& Die gesundheitskompetente Jugendinfo [Guidelines: The health literate open youth work and youth information center]. Wien: Bundesweites Netzwerk Offene Jugendarbeit (bOJA); 2016.

96. Jack BW, Paasche-Orlow M, Mitchell SA, Forsythe SR, et al. An overview of the re-engineered discharge (RED) toolkit: Agency for Healthcare Research and Quality. Rockville: Agency for Healthcare Research and Quality; 2013.

97. Lukas CV, Holmes SK, Cohen AB, Restuccia J, et al. Transformational change in health care systems: an organizational model. Health Care Manag Rev. 2007;32(4):309-20 https://doi.org/10.1097/01.HMR.0000296785.29718.5d.

98. Pelikan JM, Dietscher C. Promoting health literacy by implementing the concept of the health literate health care organization. Geneva: Geneva Global Health Literacy Forum; 2015

99. Koh HK, Brach C, Harris LM, Parchman ML. A proposed 'health literate care model' would constitute a systems approach to improving patients' engagement in care. Health Aff (Millwood). 2013;32(2):357-67 https://doi. org/10.1377/hlthaff.2012.1205.

100. Institute of Medicine. Measures of health literacy: workshop summary. Washington, DC: The National Academies Press; 2009.

101. Willis CD, Saul JE, Bitz J, Pompu K, Best A, Jackson B. Improving organizational capacity to address health literacy in public health: a rapid realist review. Public Health. 2014;128(6):515-24 https://doi.org/10.1016/j. puhe.2014.01.014

102. Argote L. Organizational learning: creating, retaining and transferring knowledge. 2nd ed. Boston: Springer; 2013. https://doi.org/10.1007/978-1-4 614-5251-5

103. US Department of Health and Human Services. Communicating Health: Priorities and Strategies for Progress. Washington, DC: US Department of Health and Human Services; 2003.

104. Wynia MK, Osborn CO. Health literacy and communication quality in health care organizations. J Health Commun. 2010;15(S2):102-15.

105. Andrulis DP, Brach C. Integrating literacy, culture, and language to improve health care quality for diverse populations. Am J Health Behav. 2007; 31(Suppl 1):122-33 https://doi.org/10.5555/ajhb.2007.31.supp.S122.

106. Pratt-Chapman M, Willis A, Masselink L. Core competencies for oncology patient navigators. J Oncol Navigation Survivorship. 2015;6:16-21.

107. Kagawa-Singer M, Dressler WW, George SM, Elwood WN. The cultural framework for health: an integrative approach for research and program design and evaluation: National Institutes of Health, Office of Behavioral and Social Sciences Research; 2014.

108. Epstein RM, Street RL Jr. Patient-centered communication in cancer care: Promoting healing and reducing suffering. Bethesda: National Cancer Institute, NIH Publication No. 07-62; 2007.

109. Agency for Healthcare Research and Quality. CAHPS Clinician \& Group Survey. 2013. https://cahps.ahrq.gov/surveys-guidance/cg/index.html. Accessed 9 Feb 2021.

110. Shoemaker SJ, Wolf MS, Brach C. Development of the patient education materials assessment tool (PEMAT): a new measure of understandability and actionability for print and audiovisual patient information. Patient Educ Couns. 2014:96(3):395-403 https://doi.org/10.1016/j.pec.2014.05.027.
111. Bax J. Quickscan Health Literacy Toolbox. Dutch Institute for Healthcare Improvement (CBO). Utrecht: Dutch Institute for Healthcare Improvement; 2014.

\section{Publisher's Note}

Springer Nature remains neutral with regard to jurisdictional claims in published maps and institutional affiliations.
Ready to submit your research? Choose BMC and benefit from:

- fast, convenient online submission

- thorough peer review by experienced researchers in your field

- rapid publication on acceptance

- support for research data, including large and complex data types

- gold Open Access which fosters wider collaboration and increased citations

- maximum visibility for your research: over $100 \mathrm{M}$ website views per year

At BMC, research is always in progress.

Learn more biomedcentral.com/submissions 